\title{
JOURNAL.RU
}

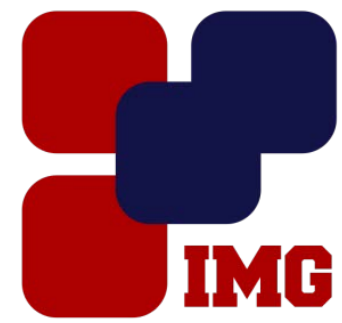
IYANOY Management GROUP

Смирнов А.В., Андрианов И.А., Суконщиков А.А., Бахтенко Е.А. Вологодский государственный университет

Вологда, Россия

doi: 10.18411/lj-31-01-2017-1-11

idsp 000001:lj-31-01-2017-1-11

\section{Математическая модель оптимизации доставки товаров автотранспортом на разветвленной сети дорог для решения задачи кластеризации}

Для заданного региона обслуживания с помощью технологии ГИС предоставляется карта автомобильных дорог, на которой указаны пункты, соответствующие источникам (поставщикам) и приемникам (получателям или потребителям) грузов (товаров). Поставщику приписан парк автотранспорта, характеризующийся количеством автомобилей определенного типа и их массогабаритными параметрами. Поставщику поступают заявки от потребителей по количеству и ассортименту товаров. Каждый вид товара характеризуется массогабаритными параметрами. Ставится задача нахождения для заданного парка автотранспорта маршрутов развозов грузов от поставщика потребителям, обеспечивающее снижение суммарных затрат на перевозку товаров.

Заявки от потребителей, являющиеся входной информацией, поступают поставщику из Торговой системы.

Матрица взаимных расстояний между объектами развоза, также поступающая на вход системы, рассчитывается средствами ГИС. Рассматривается сетка дорог с большим количеством узлов - перекрестков, тупиков и точек обслуживания, через которые должны пройти маршруты движения транспорта. Сетке дорог ставится в соответствие ориентированный граф, вершинами которого являются узлы данной сетки, а ребрами - отрезки дорог между узлами (движение по дороге может быть односторонним). Каждому ребру приписывается длина расстояние между соответствующими узлами. Ищется набор оптимальных маршрутов, начинающихся и заканчивающихся в заданных точках, и ограниченных некоторой функцией от длин ребер графа, которая может учитывать физическую длину маршрута (километраж), либо время движения транспорта, либо стоимостные характеристики маршрута движения.

Таким образом, расстояния между объектами задаются квадратной матрицей расстояний $\mathrm{A}=[\mathrm{a}(\mathrm{i}, \mathrm{j})]$ размерности $n x n$, где $\mathrm{a}(\mathrm{i}, \mathrm{j})$ - расстояние от пункта і до пункта ј. Отметим, что, в общем случае, матрица расстояний не является симметричной (одностороннее движение, сложные транспортные развязки и т.д.).

Решение поставленной задачи развоза товаров осуществляется в два этапа. 
На первом этапе решается задача разбиения региона на компактные зоны обслуживания (группирование объектов-получателей для каждого маршрута). Эту задачу будем называть задачей кластеризации. На втором этапе решается задача нахождение оптимального по заданному критерию (суммарному расстоянию, времени, стоимости доставки) порядка объезда получателей для каждого маршрута. Эту задачу будем называть задачей маршрутизации. После решения этих двух задач формируются маршруты и расписания движения для всех автомобилей. Для каждого из этих двух этапов реализовано семейство алгоритмов, позволяющих получать результат в зависимости от размерности исходной задачи, вычислительных ресурсов и требований, предъявляемых к решению.

Задача кластеризации. Задача кластеризации реализуется набором методов (алгоритмов), каждый из которых осуществляет разбиения региона на компактные зоны обслуживания.Метод основан на выборе транзитивно ближайших сообщений.

При данном подходе каждому объекту сообщению ставится в соответствие пункт на карте. Пункты характеризуются расстояниями между собой.

Пусть e = maxa(i, j) по всем i, j - максимальный элемент матрицы расстояний A.

Тогда матрица $\mathrm{B}=[\mathrm{b}(\mathrm{i}, \mathrm{j})]$ размерности $\mathrm{nxn}$, где $\mathrm{b}(\mathrm{i}, \mathrm{j})=1$ - a(i, j) / е задает нечеткое отношение сходства. $0 \leq \mathrm{b}(\mathrm{i}, \mathrm{j}) \leq 1$.

Транзитивное замыкание нечеткого отношения сходства задается матрицей $\mathrm{D}=[\mathrm{d}(\mathrm{i}, \mathrm{j})]$ размерности nхn. $0 \leq \mathrm{d}(\mathrm{i}, \mathrm{j}) \leq 1$.

$\mathrm{D}=\mathrm{B} U \mathrm{~B}^{2} \mathrm{UB}^{3} \mathrm{U} \ldots \mathrm{UB}^{\mathrm{n}}$,

где U - операция объединения нечетких отношений (МАX).

$\mathrm{B} 2$ = ВоВпредставляет собой (Max - $\min )$-композицию нечеткого отношения самого на себя.

$b^{2}(\mathrm{x}, \mathrm{z})=\operatorname{MAX}[\operatorname{MIN}(b(\mathrm{x}, \mathrm{y}), b(\mathrm{y}, \mathrm{z}))]$

$\mathrm{Bk}+1=\mathrm{BkoB}$ - (Max - min)-композиция нечетких отношений Bk и B.

$b^{\mathrm{k}+1}(\mathrm{x}, \mathrm{z})=\operatorname{MAX}\left[\operatorname{MIN}\left(b^{\mathrm{k}}(\mathrm{x}, \mathrm{y}), b(\mathrm{y}, \mathrm{z})\right)\right]$

$\mathrm{D}$ - матрица задающая нечеткое отношение эквивалентности (подобия). Это отношение рефлексивно, симметрично и транзитивно. Согласно теореме о декомпозиции для отношения подобия, для каждого значения матрицы D (в порядке возрастания их значения) получаем транзитивно ближайшие сообщения (пункты).

Таким образом, в итоге получаем разбиение множества объектов на заранее заданное число компактных (транзитивно-ближайших) групп.

Раскраска графов.

Задача кластеризации может быть сведена к задаче раскраски вершин графа. Для этого строится граф несовместимости. Вершинам графа соответствуют объекты-потребители (пункты на карте), и две вершины - смежные, если соответствующие им объекты не могут находиться в одной группе. Причины несовместимости определяются различными факторами: большое расстояние между объектами, различие заявок - объектами заказаны разные товары, и т.п.

Ставится задача раскраски вершин такого графа несовместимости при различных условиях (ограничениях, критериях), среди которых: 
- Минимальная раскраска графа (получение минимального числа компактных групп).

- Раскраска графа в заданное число красок (разбиение на группы, соответствующие парку автотранспорта).

- Раскраска графа в заданное число красок с ограничением на количество соцветных вершин (разбиение на группы, соответствующие парку автотранспорта и возможностям перевозки).

Для реализации метода используются оценки хроматического числа графа и спектр алгоритмов раскраски графов, учитывающих размерность решаемой задачи и отведенное на решение время.

Частотно-матричный метод.Подход к постановке задачи аналогичен предыдущему, но в качестве исходной модели рассматривается матрица инциденцийQ $=[q(\mathrm{i}, \mathrm{j})]$. Столбцам матрицы соответствуют, буквы - объекты (пункты на карте), строкам - подмножество, определяемое словесным отношением Si, i=1, 2, .., n, и q(i, j) = 1, если в i-е слово входит j-я буква, и q(i, j) = 0 - в противном случае.

Построение матрицы инциденций осуществляется таким образом, что каждое словесное отношение, определяется выполнением условия совместности (несовместности) вхождения соответствующих объектов в одну группу.

На основе построенной матрицы определяется частотная матрица отношений $\mathrm{F}=\mathrm{QTxQ}$, элементы которой $\mathrm{f}(\mathrm{i}, \mathrm{j})$ используются для целенаправленного нахождения ближайших объектов и включения их в одну группу. В основе данного подхода лежит аппарат дифференцирования дискретных моделей.

Алгоритм учитывает заявки, поступившие от потребителей.

Исходной моделью является матрица расстояний $\mathrm{A}=[\mathrm{a}(\mathrm{i}, \mathrm{j})]$ размерности nxn, где a(i, j) - расстояние от пункта i до пункта j.

Ищется самая удаленная точка (пункт) и к ней последовательно добавляются ближайшие с учетом роста группы. В матрице А ищется строка, сумма элементов которой максимальна. Соответствующий ей пункт вносится в группу. i1 - координата строки.

Ищется ближайший пункт к внесенному в решение - минимальный элемент матрицы А в столбце i1. Соответствующий ей пункт вносится в группу. i2 координата строки.

Ищется пункт ближайший к внесенным в решение - минимальная сумма элементов матрицы А в столбцах і1 и i2. Соответствующий ей пункт вносится в группу. i3 - координата строки.

И так далее. На очередном шаге $\mathrm{k}$ ищется пункт ближайший к внесенным в решение $\mathrm{k}$ пунктам i1, i2, i3..., ik - это минимальная сумма элементов матрицы А в указанных столбцах.

Процесс завершается, если величина заявки в очередной точке (пункте) $\mathrm{ik}+1$ больше остатка rest, равного разности между максимально возможной величиной суммарных заявок (загрузка авто средства) и суммой заявок от всех включенных в решение пунктов i1, i2, i3..., ik.

В зависимости от порядка организации перевозок пункт ik+1 может быть внесен в решение с числом выполненных заявок rest. В этом случае повышается общая загрузка авто средств, но пункт, будет посещаться неоднократно. 